\title{
Espacios online educativos a partir de la percepción de la propia experiencia de estudiantes universitarias/os
}

\begin{abstract}
Resumen: Este artículo analiza las maneras en que estudiantes de carreras en modalidad online perciben y experimentan los ambientes virtuales que habitan y transitan, como así también sus posibilidades de agencia y de expresión, y las percepciones respecto de las y los demás actores. Primeramente, se presenta una sistematización teórica sobre los ambientes virtuales como espacios sociales y educativos, ya sean aulas virtuales institucionales o plataformas de redes sociales. A continuación, se analizan indicadores basados en datos cuantitativos producidos ad hoc, y finalmente, se presenta una sistematización de líneas derivadas del análisis para la construcción de espacios educativos online inclusivos y democráticos, que intenten no repetir desigualdades históricas y contemplen la diversidad de experiencias y agencias. Metodológicamente, se combinó la producción de datos cuantitativos con análisis cualitativo basado en el método descriptivo y analítico. Los datos fueron producidos en base a una encuesta a ingresantes de carreras online en la Universidad (Nombre omitido).
\end{abstract}

Palabras-clave: Espacios virtuales educativos. Redes sociales. Aulas online. Comunicación/Educación. Agencia.

Introducción

No cabe duda de que la popularización de las estrategias y demandas de inserción de tecnologías digitales en los procesos educativos es una tendencia marcadamente reconocible durante las últimas décadas en América Latina en los diferentes niveles académicos.

De la mano de políticas públicas, programas de organismos internacionales, acciones oficiales locales y municipales, alianzas con sectores privados $\mathrm{y}$, menos frecuentemente, con la sociedad civil, la naturalización de la idea de que las tecnologías digitales son agentes que necesariamente deben ser integrados a las prácticas educativas se instaló con mayor o menor éxito en los imaginarios contemporáneos. Claramente, los imaginarios por definición raramente toman como centro de su representación a las experiencias periféricas o diferenciales, siendo en general las experiencias centrales, urbanas, metropolitanas y de clase media, los modelos que se imponen como ejemplos o como patrones a partir de los cuales se entiende, significa y gestiona la experiencia general.
Verónica Sofia Ficoseco Universidade Nacional de la Patagonia Austral PPGE - UFBA vsficoseco@gmail.com Melina Daniela Gaona Universidad Nacional de Quilmes CEHCMe - CONICET melina.d.gaona@gmail.com 
(1) Las medidas de aislamiento preventivo y distanciamiento social se encuentran vigentes en el conjunto del territorio nacional a la fecha de cierre de este artículo, con variaciones regionales de acuerdo con la incidencia de los casos.
En este contexto, son innumerables los trabajos desde las investigaciones en el área de ciencias de la educación que alertan sobre la necesidad de realizar estudios locales y situados que permitan comprender y gestionar de modo adecuado y sensible las experiencias con tecnologías digitales en cada contexto educativo, que señalan la importancia de trascender los enfoques instrumentalistas de las tecnologías en el aula y favorecer la apropiación creativa, que alertan sobre las consecuencias y limitaciones que pueden devenir de la falta de agencia y control ciudadano sobre los sistemas tecnológicos que moldean y atraviesan nuestras vidas cotidianas, no sólo en el ámbito educativo, y, finalmente, que desarrollan propuestas científicamente sustentadas para el trabajo curricular y pedagógico que permita la inclusión y apropiación crítica. (PRETTO, 2017)

Dentro de los debates sobre las apropiaciones tecnológicas en las prácticas educativas contemporáneas se ubica una línea que en la actualidad podría considerarse central: los ambientes virtuales de aprendizaje, que incluyen tanto a los ambientes institucionales de educación a distancia o campus virtuales, como a la instrumentalización de ambientes online privados o comerciales, como, por ejemplo, las plataformas de redes sociales.

Tanto los datos de este artículo, como su escritura, se dan en el marco del actual contexto de pandemia de Covid-19, cuya dispersión en el hemisferio norte comenzamos a conocer a través de los medios de comunicación y las redes digitales desde fines del año 2019, antes del registro de los primeros casos en América Latina en febrero de 2020. En Argentina, la adopción temprana de medidas oficiales de aislamiento social preventivo y obligatorio a

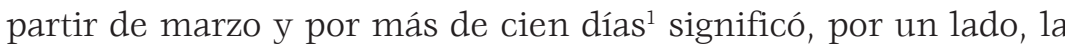
posibilidad de mantener niveles relativamente bajos en relación con los países de la región en cuanto a la transmisión local del virus y preservación de la capacidad funcional del sistema público de atención de salud. Por otro lado, implicó la necesidad de trasladar a la virtualidad, o a la mediación tecnológica, muchas de las actividades administrativas, comerciales, políticas, burocráticas, culturales y educativas que tradicionalmente se realizaban de forma presencial.

Al mismo tiempo que esta situación demandó adaptaciones y activación de estrategias creativas para cada sector, también dejó al descubierto profundas desigualdades en el acceso a servicios 
básicos de conectividad y disponibilidad de equipamientos, y en la capacitación de actores/as del sector público y privado para encarar las mudanzas en las rutinas de producción. En lo que compete a la educación, recrudeció los debates sobre la formación docente y las demandas de larga data acerca de la discusión sistémica de actualizaciones curriculares, la dotación de equipamientos y conectividades de calidad y la capacitación general de actores docentes y no docentes con visión de largo plazo, no exclusivamente en base a iniciativas dispersas y efímeras. (BIANCHI, SANDOVAL, 2020; KAPLÚN, 2020)

En lo que refiere específicamente a las universidades, en medio de la consigna de celeridad dictada por la emergencia, instituciones públicas y privadas se apresuraron a pensar, dentro de sus posibilidades y en base al ejercicio de la autonomía de gestión, estrategias para paliar la situación. En general, esto decantó en la implementación de sistemas de educación a distancia por diferentes vías, que van desde las propuestas de educación cien por ciento virtual hasta las diferentes escalas de la multimodalidad con mayor protagonismo de instancias presenciales combinadas con apoyos remotos. En lo que respecta a las carreras y cursos, se han implementado, desde acompañamientos pedagógicos guiados por lecturas y diálogos online, a ofertas académicas alternativas para el cursado de créditos especialmente formulados para el contexto de aislamiento actual hasta la reanudación de las cátedras presenciales, pasando por el traslado inmediato y provisorio de todas las materias y créditos presenciales para el dictado obligatorio online o bimodal. Entre las universidades públicas se dirime también como argumento a favor de las medidas emergenciales la necesidad de continuar garantizando el derecho al acceso a la educación superior pública y gratuita, argumento que atraviesa fuertemente las deliberaciones sobre la adopción de diferentes versiones y gradaciones de la educación en entornos virtuales. Ya sean estos los campus virtuales gestionados por las universidades o los grupos o espacios en plataformas de redes sociales creados $\mathrm{y}$ gestionados por docentes y estudiantes como espacios para las prácticas educativas cotidianas.

En el caso de universidades públicas que ya contaban con oferta académica sólida en modalidad virtual, con equipos docentes específicos para dicha tarea y con plataformas o campus virtuales maduros y consolidados, el contexto de situación implicó la 
intensificación de los usos de dichos espacios, la migración de instancias tradicionalmente presenciales de la vida institucional hacia la plataforma virtual, y la adaptación de cursos presenciales para ser alojados en los campus virtuales. Es el caso de la Universidad, Unidad Académica San Julián de la Universidad Nacional de la Patagonia Austral (UASJ UNPA), que será referencia empírica de este trabajo. (UNPA,2020) En la contingencia sanitaria y política global que pone en tensión las prácticas cotidianas de formación, las mediaciones y los intercambios entre pares en estos ámbitos, consideramos fundamental construir información de carácter específico y situado en el cruce espacios educativos, virtualidad y experiencia. Este trabajo busca ser un aporte a dichas discusiones.

Desde el punto de vista de la comunicación/educación, de los estudios de género y del construccionismo social de las tecnologías, la comprensión de los sistemas de educación virtual o a distancia se basa en la comprensión integral de dichos sistemas en tanto espacios, es decir, como estructuras sociales, ámbitos de relaciones cuya unidad analítica son las interacciones y los agentes que las conforman. De este modo, la atención a los espacios y los contextos específicos en que las prácticas online se inscriben y se desarrollan, al mismo tiempo que a las posiciones que cada agente ocupa en dichas dinámicas y sus posibilidades de agencia, son factores centrales para el análisis, para la comprensión crítica y para la planificación sensible de ambientes educativos democráticos. (FICOSECO, 2018)

La pregunta por la experiencia de educación en entornos virtuales es una pregunta que también debe atender a las condiciones materiales en las que se libran tales tránsitos. De entre el cúmulo de elementos que conforman y dan sentido a esas condiciones, inicialmente procuramos atender a la materia espacial virtual entre distintos ámbitos o lugares en los que se dirimen los caminos del vínculo en el proceso de formación. Pero además nos interesa reconstruir cómo perciben las y los sujetos esos distintos ámbitos, su involucramiento, sus capacidades y sus relaciones, a la luz del interés por procurar una comprensión integral de la educación mediada en estas condiciones.

El objetivo de este artículo es analizar críticamente las maneras en que personas que cursan carreras en modalidad online perciben y experimentan los ambientes virtuales que habitan y transitan, como así también sus posibilidades de agencia y de expresión y 
las de los demás actores en cada uno de ellos. Para ello, primero se presenta una propuesta de abordaje teórico analítica de los ambientes virtuales de aprendizaje en tanto espacios sociales y educativos, mediante una selección de líneas conceptuales actualizadas acerca de los espacios virtuales inscriptas en la perspectiva sociotécnica, el construccionismo social de las tecnologías y los estudios feministas. A continuación, se analizan algunos indicadores basados en datos cuantitativos producidos ad hoc mediante una encuesta aplicada a ingresantes de carreras que se dictan totalmente online en la Universidad (UNPA). Finalmente, se presentan algunas propuestas o líneas centrales derivadas del análisis que puedan conducir aportes a las reflexiones sobre la construcción de espacios educativos online inclusivos y democráticos, que intenten no repetir desigualdades históricas y contemplen la diversidad de experiencias y agencias, ya sean estos espacios las aulas virtuales de un campus universitario o los grupos en plataformas de redes sociales instrumentados por docentes y estudiantes para sus prácticas educativas cotidianas.

\section{Los ambientes virtuales como espacios en continuum: campus institucionales y plataformas de redes sociales}

Teniendo en cuenta los postulados de la geografía feminista, es posible sostener que los ambientes u entornos virtuales son espacios, son construcciones espaciales. (MASSEY, 2005) Esta línea teórica considera a los espacios como productos de las interrelaciones, es decir, como una dimensión social constituida a partir de esas interrelaciones. Por lo tanto, son sólo abordables o analizables a partir de la atención a esas relaciones. Esta visión materialista del espacio entiende que las relaciones que lo constituyen son inseparables de las prácticas que efectivamente se terminan dando y transcurriendo en ellos. Por ello se considera que se encuentran siempre en proceso de construcción.

El espacio es, desde esta definición, tanto un objeto como un proceso, de manera simultánea. Como objeto, es posible considerar que para cualquier situación social, la estructura objetiva y material en que ocurren las interacciones es el espacio con el que los agentes se encuentran. En tanto al espacio como proceso, toma la forma de lo que habitualmente es definido como habitus, es decir, que contempla tanto el modo en que corporizamos o 
habitamos el espacio, como también el modo en que lo clasificamos y reconocemos.

Una perspectiva que va más allá de esta primera aproximación se abre con la incorporación de la definición de trialéctica del espacio. (SOJA, 1996) Este concepto, traído de los postulados de Lefebvre (2013) comprende una división terminológica y analítica de las distintas instancias que conforman toda espacialidad. Inicialmente, una primera versión del espacio que sería la "práctica espacial", el espacio percibido, producido y reproducido materialmente en la cotidianidad. El espacio resultado de la actividad humana. Una segundo escala de la espacialidad comprende a las "representaciones del espacio", o el espacio concebido. Es la planificación atada a las relaciones de producción, el espacio reglado y congruente con las representaciones del poder y las ideologías dominantes. Por último, Soja (1996) comprende que existe un tercer espacio que escapa del binarismo entre la estructura y la reproducción, que tiene que ver con los "espacios de representación", resultado del espacio vivido. Esta instancia implica las apropiaciones dichas y no dichas, la contracara combinada de los otros dos espacios, y lo vivido entre medio de ellos. Es una instancia de apropiación que habilita la resistencia, la modificación y la trasgresión.

Otra definición en torno a la espacialidad que resulta particularmente productiva para pensar los espacios virtuales, tiene que ver con considerarlos siempre en un estado presente, tanto en sus versiones actuales, como en las virtuales. En este punto se abren reflexiones sobre los espacios virtuales como una acumulación histórica del pasado, lo cual implica considerar el modo en el que intervienen en su constitución las posiciones históricas de cada agente que lo habita y transita. En este sentido, es probable que las desigualdades y opresiones históricas se repliquen o se renueven en instancias espaciales virtuales en base a las posiciones diferenciales que marcan las trayectorias de los sujetos/as y sus posibilidades de agencia y resistencia. (COUTO, 2017; FICOSECO, 2018) Se trata de una instancia frecuentemente poco explorada cuando se enfoca analíticamente en los espacios virtuales, dado que suelen recibir los motes de lo efímero, lo fugaz, lo inmaterial, o hasta de lo no relevante o inexistente, ocluyendo de algún modo la posibilidad de visibilizar su eficacia como instancia en que las relaciones sociales cotidianas se dirimen. (BUMACHAR; FERREIRA, 2018) 
Para pensar a las espacialidades online o mediadas tecnológicamente, resulta productivo articular una línea conceptual enfocada en comprender los vínculos que se produce en los entornos virtuales; enfocada en las experiencias en ambientes virtuales, atendiendo a las articulaciones entre presencia virtual y presencia física. En este sentido, resulta de interés discutir las instancias por las cuales comprendemos que las corporalidades y las espacialidades virtuales serían elementos tanto co-constitutivos como relacionales, atendiendo a la entidad simbólica que tienen ambas presencias.

Por lo general, cuando se enfocan analíticamente los espacios educativos en la virtualidad suelen tomarse como norma los entornos virtuales institucionales, los llamados campus virtuales o AVA. Sin embargo, dado el tenor de continuum que caracteriza a las experiencia, sean estas presenciales o medidas tecnológicamente, es necesario pensar a la experiencia virtual también en un continuum entre ámbitos que se transitan online de manera cotidiana. La incorporación analítica de los ámbitos virtuales privados o comerciales, como las plataformas de redes sociales, hace parte del análisis para reflexionar sobre las prácticas educativas online, dado ese carácter de continuum en la experiencia online. Esto complejiza un acercamiento sobre la experiencia educativa al considerar los distintos tenores esperables entre espacios producidos con distintos intereses y tránsitos variables de acuerdo con las presencias múltiples en cada uno.

Esta exploración en ambos registros resulta necesaria si se pretende comprender de modo integral la configuración de experiencias online. Las personas indefectiblemente configuran sus modos de habitar, de transitar y de agenciar online tanto en espacios institucionales, como podrían ser las plataformas universitarias o campus online, como los espacios privados y comerciales, como las plataformas de redes sociales. Esto se da de modo conjunto y en base a lo que suponen las experiencias materiales con tecnologías digitales o, más bien, en base a las posibilidades y configuraciones de esas experiencias digitales en la práctica efectiva.

Más allá de contemplar esta continuidad, es indispensable atender a que cada modo de producción tiene su propio espacio característico. Por lo que los entornos comerciales terminan inmanentemente siendo dispuestos en términos diferenciados respecto de los espacios institucionales. Esto es perceptible tanto en la circulación permitida para usuarios/as y los recursos visuales 
e interactivos disponibles para ello, como en experiencias que se transitan de forma aparentemente más amigable en los entornos comerciales gracias al cuidadoso diseño de experiencia del usuario. Pero también cuando percibimos experiencias o versiones de los espacios más restrictivas o que tienden a las fórmulas privativas y excluyentes -cuando no abiertamente expulsivas de quien se considera diferente- de los espacios, o con la experiencia de las burbujas informativas basadas en la selección de contenidos que serán visualizados por cada usuario según sus coincidencias ideológicas y ocluyendo la visibilización de lo diferente en los tránsitos cotidianos, o las estructuras empresariales emblemáticas del nuevo capitalismo basadas en el extractivismo de datos. (SILVEIRA, 2019) Todas estas facetas deben ser tenidas en cuenta como parte de los propósitos con los que dichos espacios fueron inicialmente generados y por tanto como elementos presentes en cualquier apropiación o tránsito.

En base a lo hasta aquí expuesto, podemos establecer que los espacios virtuales no son todos iguales, sino que las condiciones en que se disponibilizan y las opciones que ofrecen para ser habitados y transitados pueden ser muy diversas, ya sea que se trate de ambientes institucionales o de plataformas de redes sociales. Establecemos también que dado que son las interacciones y las relaciones entre personas los elementos constitutivos de las dimensiones espaciales, cualquier limitación o elemento que administre los tránsitos y apropiaciones de la dimensión objetiva de esos ámbitos necesariamente influirá y será influenciado por las interacciones que allí se terminen construyendo. En el mismo sentido, también las condiciones históricas de cada sujeto/a serán estructurantes de esas dinámicas y factores que moldean las posibilidades de apropiación y de tránsito por esos espacios. De allí la pertinencia de comprender, cuando hablamos de ambientes virtuales, la diversidad de condiciones y de posibilidades de cada una de las versiones de las espacialidades online. En el caso de ambientes online específicamente construidos para prácticas educativas, resulta urgente la atención a esta diversidad de elementos, a las diferentes posibilidades y apropiaciones implicadas en cada ámbito, a las accesibilidades y posibles agencias disponibles para unos y otras, bajo el objetivo de la construcción de ámbitos de comunicación/educación inclusivos y democráticos. 


\section{Las experiencias virtuales}

Como se mencionó anteriormente, el espacio es abordable o visible a partir de las experiencias que allí se desarrollan. Este enfoque supone entender a la experiencia siempre como la intersección en la que se entraman condiciones materiales, simbólicas y las posibilidades de articulación o de reconocimiento, y la faceta de la narrativa de dichas condiciones. (DELFINO, 1998; GAONA, 2017)

Al considerar la experiencia en clave interseccional, desde las teorías construccionistas de las tecnologías y en clave de género, desde el enfoque que venimos desarrollando entendemos que se hace necesario reconstruir la serie de condiciones culturales que delimitan, corporizan y habilitan posibilidades restringidas a las expectativas de socialización de cada persona en esos espacios.

Lo mencionado implica reconocer que la experiencia diferenciada, construida desde corporalidades otras o diversas, deviene en una experiencia desigual para muchas personas, que supone límites, opresiones y ajenidad, al mismo tiempo que también habilita posibilidades de reconfiguración y resistencia. Por ello se atiende a la configuración de una perspectiva que contemple tanto la dimensión de la experiencia habitada, o sea la experiencia corpórea en sí misma, como al carácter epistemológico de la experiencia.

Sobre este último punto, sostenemos que la experiencia en general, y la experiencia virtual en particular, se constituye en base a situaciones, posiciones y materialidades específicas, desde esos lugares y posicionamientos siempre particulares, siempre marcados históricamente, y siempre en torno de las relaciones vigentes de poder que son los marcadores a partir de los cuales los sujetos/as se apropian de ciertas categorías y de ciertos relatos disponibles para poder nombrar al mundo. En este punto se abre una faceta por la cual en esta posibilidad de transformación inherente a la experiencia encontramos también modos por los cuales la experiencia habilita renombrar ciertas prácticas, ciertas interpretaciones de lo real e inclusive cuestionarlas.

Es en esta confluencia conceptual en que ubicamos las concepciones tanto de los espacios virtuales como de las experiencias que los constituyen, y por tanto las percepciones, representaciones y modos de construir agencias y repertorios para nombrar los mismos desde los tránsitos alternativos de los y las sujetos/as históricamente situados/as. A partir de allí, habilitamos una instancia 
analítica que busca la construcción legitimada y factible de formas de ser colectiva de la experiencia en los tránsitos virtuales por parte de sujetos/as que construyen experiencias diferenciadas en esos espacios construidos.

\section{Espacios virtuales y educación}

Desde el punto de vista de los espacios virtuales $u$ ambientes online instrumentados para la realización de actividades educativas de manera mediada, adoptamos la definición propuesta desde los estudios sociotécnicos, que enfocan a estos ambientes en tanto redes de elementos, actores, artefactos y sus condiciones materiales situadas interactuando en un momento histórico y un contexto determinado. Una operacionalización del concepto de entorno sociotécnico particularmente interesante para este trabajo es el concepto de dispositivo hipermedial dinámico, definido como una red sociotécnica de carácter participativo y no excluyente, construido con fines educativos, investigativos y/o laborales en un contexto institucional situado. (SAN MARTÍN; GUARNIERI; BONGIOVANI, 2014) Pensando desde la educación a distancia, esas características están presentes en la mayor parte de las propuestas y sistemas educacionales en dicha modalidad, por lo menos mencionadas entre sus intenciones.

En base a esto, y siguiendo la perspectiva presentada hasta aquí, entendemos que los entornos virtuales educativos pueden ser estudiados en tanto espacios, es decir, son considerados espacios sociales, construidos, disputados, atravesados por tensiones, habitados, percibidos diferencialmente según el lugar que cada actor ocupa en ellos y con normas específicas que regulan su funcionamiento y tránsitos posibles, al mismo tiempo que proporcionan los márgenes necesarios para la resistencia y replanteo de dichos límites.

Entonces, las interacciones entre las personas en estos ambientes se configuran también en esos espacios al mismo tiempo que son configuradas por ellos. Las relaciones interpersonales que se establecen en espacios educativos institucionales online y en plataformas de redes sociales digitales, poseen diferencias cualitativas en los modos en que éstas son valoradas y experimentadas, según lo han observado diversas investigaciones. Por un lado, con respecto del involucramiento o el compromiso personal que puede esperarse cuando se trata de actividades o intercambios 
desarrollados en estas plataformas. Desde algunas perspectivas se ha señalado que la presencia y las relaciones online son valoradas y consideradas en el mismo nivel de compromiso que cualquier otra actividad cotidiana presencial, sin por ello dejar de ser percibidas diferencialmente. (SIBILIA, 2019; TURKLE, 2012) En cuanto a las dinámicas sociales online con fines educativos, algunos estudios propios indican que las habilidades digitales o habilidades para el manejo técnico, la expresión y la comunicación en espacios online, son el elemento central para definir el involucramiento y la fluidez de las dinámicas de comunicación entre docentes y estudiantes. (FICOSECO; CEVALLOS; OVIEDO, 2019)

En base a estas observaciones, diversos estudios han señalado que las redes sociales digitales como ambientes de interacción pueden ser apropiadas y moldeadas por los diferentes grupos y comunidades, por ejemplo para la construcción de procesos comunicacionales con fines educativos y formativos. Sin embargo su instrumentación no permite dar por sentada de antemano la existencia de posibilidades de agencia similares para todos/as los/ as participantes, por lo que resulta fundamental tener en cuenta diversos factores generacionales, económicos, culturales, raciales, de sexualidad y de género, por mencionar algunas, en las planificaciones e intervenciones educativas mediadas. (FICOSECO, 2018)

\section{Metodología}

Para el presente artículo se combinaron instancias de investigación cuantitativa y cualitativa. En primer lugar, para la producción de datos, se llevó a cabo un relevamiento mediante encuestas aplicadas a estudiantes que cursan carreras de dictado cien por ciento online en la Universidad (UNPA), ingresantes en el año 2020. Las carreras que se dictan en esta modalidad son tres: Licenciatura en Trabajo Social, Licenciatura en Turismo y Tecnicatura en Recursos Naturales Renovables, siendo en total 91 los ingresantes matriculados en el año 2020. El formulario de encuesta se dispuso online en un aula del entorno virtual institucional accesible para ingresantes de estas tres carreras, o sea para estudiantes matriculados en las carreras de modalidad virtual. La participación fue voluntaria y anónima. Se recibieron en total 33 respuestas.

Respecto del instrumento de recolección de datos, la encuesta se articuló en tres secciones: percepción de las propias habilidades 
tecnológicas, experiencias con plataformas de redes sociales y experiencias con el entorno virtual educativo de la universidad. El formato de las respuestas fue en escala de Likert para las variables continuas y escala de dos opciones para las variables discretas.

Por otra parte, se utilizó el método cualitativo de análisis crítico y descriptivo (MINAYO, 2011), articulado en base a la construcción de categorías centrales. Estas categorías centrales fueron formuladas en base a resultados preliminares de estudios cualitativos realizados en el mismo contexto, constituyeron la base de las tres secciones en que se divide el instrumento de producción de datos (encuesta), en este artículo se analizan dos de esos ejes: las experiencias en redes sociales y las experiencias en el entorno educativo universitario.

Estos dos ejes a su vez fueron transversalizados analíticamente por las categorías teóricas del espacio y la experiencia. De ese modo, se configuró un análisis que indaga, por un lado, en los tránsitos que las personas configuran en los ambientes online como continuum, $\mathrm{y}$, por otro lado, en las relaciones que se establecen, posibilidades de agencia y de auto expresión que se perciben.

\section{Percepciones sobre los espacios online}

Dado que el grupo analizado se compone de estudiantes de carreras de dictado cien por ciento virtual, sabemos de antemano que todos ellos/as son usuarios/as de la plataforma universitaria de la Universidad (UNPA). En cuanto a los usos de redes sociales, el 97\% de los encuestados manifestó ser usuario/a de una o más plataformas, siendo la mayormente preferida Whatsapp $(63,3 \%)$, seguida por Facebook (24,2\%) e Instagram (9,1\%). Por tanto podemos asegurar que se trata de un grupo que mantiene intensos y estables tránsitos online.

Respecto de la elaboración de la presencia online, casi la mitad $(48,4 \%)$ asegura que mantiene actualizado su perfil e información online, mientras que el 30\% no lo hace con tanta frecuencia, restando solo un $20 \%$ que no actualiza periódicamente su información online ni perfiles. En el mismo sentido, referido a la dedicación como a la percepción de las habilidades propias para transitar de modo seguro las plataformas online, más del 75\% de los/as encuestados/as señala que sabe cómo gestionar su información en redes sociales y se siente apropiado/a para elegir los tipos de información que comparte, y restringir con quien hacerlo. 
En cuanto a la instrumentación de esos tránsitos en plataformas de redes sociales como espacios para las actividades cotidianas, la mitad de las personas señala que utiliza las redes sociales con fines laborales o de negocios, mientras que, al ser indagados sobre los usos educativos, incluyendo buscar información para las tareas académicas y el contacto con compañeros/as de estudio y docentes, ese porcentaje asciende al $94 \%$.

En el mismo sentido, en lo referente a los tránsitos construidos en el entorno virtual universitario, sólo el 15\% señala que participa con frecuencia en foros y actividades online no obligatorias propuestas en las aulas virtuales. Esta observación de una aparente no afinidad o no preferencia por mantener interacciones en los entornos educativos, a menos que estos sean obligatorios, se ve reforzada o reafirmada cuando constatamos que el 36,4\% de los/as estudiantes/as prefiere contactarse con los/as docentes por otros medios (email o redes sociales), y no a través de la plataforma institucional. Ese porcentaje asciende al 57,6\% cuando se trata de contacto con otros/as estudiantes.

Esto permite inferir, en coincidencia con las líneas conceptuales presentadas, que tanto los espacios virtuales como las posiciones que cada uno/a ocupa en esas dinámicas mediadas son efectivamente percibidos de manera diferencial. Por decirlo de un modo más claro, no da igual un ambiente de red social o un aula virtual en el sistema institucional, como tampoco son simétricas las posibilidades de expresión que se perciben como posibles para todos/as y las que efectivamente se entiende como propias. En fundamental resaltar que no estamos refiriendo a diferencias en las condiciones tecnológicas (diferencias de diseño entre un ambiente y otro), sino que los datos aquí analizados refieren explícitamente a la percepción de diferencias en el sentido de la práctica de la interacción, de la comunicación y de la apropiación.

\section{Percepciones sobre las relaciones y las agencias online}

En lo referente a las maneras en que las personas perciben las posibilidades de agencias y relaciones en ambientes online -tanto entornos institucionales educativos como entornos comerciales-, esto se exploró, en primer lugar, a partir de la indagación acerca del grado de conocimiento sobre la existencia de límites o dinámicas qua actúan como límites para la apropiación plena de los 
espacios virtuales por parte de otros actores/as o de sí mismos/as. Por ejemplo, con las situaciones de violencia online.

Respecto de este eje, el 90,9\% de los/as estudiantes señalan que conocen o tuvieron información sobre casos de violencia o discriminación en redes sociales, mientras que, al ser indagados sobre acontecimientos análogos en las aulas virtuales de la universidad, solo el $11 \%$ expresó conocer algún caso de personas violentadas o silenciadas en esos ámbitos.

Cuando la pregunta refiere a la propia experiencia, es decir, a haberse sentido o percibido como víctima de discriminación o maltratos en un aula virtual de la universidad, el 3\% señala que fue víctima de situaciones como las mencionadas, mientras que 18,2\% se mantiene neutral, ni afirmando ni negando esa posibilidad. Mientras que, en la misma escala perceptiva, el 78,8\% de los/ as estudiantes afirman que no percibieron ese tipo de dinámicas durante sus tránsitos en los entornos universitarios online.

Las respuestas cambian notablemente cuando se contrastan los niveles de aceptación de la misma afirmación, pero en lo referente a las plataformas de redes sociales. En este caso, el porcentaje de estudiantes que afirma haber sido víctima de descalificación y discriminación en redes sociales asciende al 24,2\%. Adicionalmente, ante la pregunta sobre haber sido objeto de violencia a través de la tecnología en alguna oportunidad ya sea a través de mensajes de texto, redes sociales o email, el 27,3\% contestó afirmativamente.

Respecto de las posibilidades de expresión personal y de exteriorización de las opiniones en espacios online, el 72,7\% de los/as estudiantes señaló que concuerda con la idea de que en las redes sociales todas las personas tienen las mismas posibilidades de expresarse libremente, mientas que en lo referente a las aulas virtuales ese porcentaje fue del 75,5\%. Esto permite afirmar que, en mayor o menor medida, la mayoría de las personas incluidas en el grupo analizado perciben a los espacios virtuales como entornos de libre tránsito y expresión.

Sin embargo, ese porcentaje de coincidencia con la afirmación sobre la libertad de expresión decrece cuando se trata de uno mismo, es decir, cuando se indaga sobre la percepción sobre la propia posibilidad de expresarse libremente. En este caso, el porcentaje de estudiantes que concordó con que se siente libre para expresarse plenamente en el entorno virtual universitario cayó al 60,6\%, y en entornos de redes sociales al 57,5\%. Resulta interesante observar 
el contraste entre las percepciones de libertad y posibilidades de agencia y de expresión libre en los diferentes ambientes online. De acuerdo con lo observado, los/as estudiantes identifican un margen de libertad levemente mayor en favor de los entornos institucionales online en detrimento de las plataformas de redes sociales donde el margen de agencia percibido es menor. Por otra parte, parecen concordar en mayor medida con la afirmación extendida sobre los ambientes online como espacios de libre tránsito y agencia para todos y todas, expresando que dichos márgenes de autonomía y agencia se ven reducidos -al menos en lo que hace a su percepción- cuando se trata de las propias posibilidades de expresión.

Entonces, partiendo de la idea de que las personas entienden de manera diferencial a los espacios, de que la percepción de las propias posibilidades de agencia no es simétrica con la aceptación de las posibilidades abiertas para los otros/as, un diseño o propuesta pedagógica que apunte a integrar esos ambientes debería tener en cuenta en la base de su formulación estas características constitutivas a la hora de elegir los ambientes online, combinarlos, habilitar espacios de intercambio, visibilización, pensar estrategias de comunicación/educación que apunten a la construcción de ámbitos de agencia, participación y no exclusión.

\section{Algunas conclusiones}

El propósito de este tipo de propuestas analíticas, centradas en la comprensión de los espacios online como continuum, específicamente pensando en las potencialidades, tanto de los entornos virtuales institucionales, como de las plataformas de redes sociales para ser instrumentados como espacios online educativos, es proporcionar algunos posibles enfoques para pensar y gestionar nuevas experiencias educativas en contextos de anormalidad como el que atraviesa el presente global. Sin detrimento de que la incorporación, apropiación y configuración activa e inclusiva de esas espacialidades mediadas en las prácticas educativas contemporáneas resultaría beneficiosa no sólo en la coyuntura, sino pensando en apropiaciones democráticas, diversas y activas de esos espacios sociales por parte de prácticas educativas liberadoras.

De acuerdo con los datos analizados, pudimos observar que el grupo delimitado para este estudio se compone de personas que 
transitan de manera activa e intensa los entornos virtuales como continuum, pasando de los ambientes de aulas virtuales a las plataformas de redes sociales. Se auto definen en su mayoría como hábiles y conocedores de los condicionamientos, limitaciones, posibilidades y peligros de esos ambientes. También pudimos establecer que existe una preferencia marcada por la interacción en plataformas de redes sociales. Así, los/as estudiantes entretejen los contactos y prácticas del entorno institucional, por ejemplo, las consultas a docentes o la búsqueda de información académica, desplazándolas al ámbito de las redes sociales. Este punto permite vislumbrar la posibilidad de nuevos análisis que profundicen específicamente en los elementos comunicacionales que caracterizan unas y otras posibilidades de interacción.

Siguiendo con la línea de la percepción diferenciada entre entornos virtuales institucionales y comerciales, pudimos observar que la mayoría de los y las estudiantes valoran a los espacios virtuales institucionales como más seguros, o menos propicios al accionar violento y -detalle no menor- a la subrepticia descalificación o discriminación que lleva al silenciamiento, mientras que se percibe una mayor medida de estas problemáticas en los ambientes de redes sociales. También fue posible establecer que los y las estudiantes en su mayoría conciben a los espacios online como ambientes en los que es posible ejercer la libertad de expresión y la agencia. Notablemente, esa percepción se manifestó con menos fuerza cuando se trata de la percepción de sí mismo/a como agente con posibilidades de libre expresión y agencia.

A partir de las observaciones presentadas, resaltamos el valor de la producción de datos situados y locales para la inclusión tecnológica y de entornos virtuales en los contextos educativos. Entendemos que estas reflexiones sobre datos empíricos para pensar las prácticas educativas y también para la reflexión desde el punto de vista de la educación resulta necesaria, dado que la configuración de espacios propicios para la expresión y la agencia, la creatividad y la autonomía, resulta fundamental para cualquier praxis educativa democrática. 


\title{
Espaços educativos on-line a partir da percepção da própria experiência de estudantes universitários
}

Resumo: Este artigo analisa as maneiras pelas quais os estudantes de cursos on-line percebem e experimentam os ambientes virtuais em que habitam e transitam, bem como suas possibilidades de agência e expressão e as percepções respeito de outros atores. Primeiramente, é apresentada uma sistematização teórica de ambientes virtuais como espaços sociais e educacionais, sejam salas de aula on-line institucionais ou plataformas de redes sociais. A seguir, são analisados indicadores baseados em dados quantitativos produzidos ad hoc e, por fim, é apresentada uma sistematização de linhas derivadas da análise para a construção de espaços educacionais on-line inclusivos e democráticos, que visem não repetir as desigualdades históricas e contemplar a diversidade de experiências e agências. Metodologicamente, a produção de dados quantitativos foi combinada com a análise qualitativa, com base no método descritivo e analítico. Os dados foram produzidos a través de um questionário a estudantes de cursos on-line na Universidade (Nome omitido).

Palavras-chave: Ambientes virtuais educativos. Redes sociais. Aulas on-line. Comunicação/Educação. Agencia.

\section{Online educational spaces based on the perception of university students' own experience}

\begin{abstract}
This article analyzes the perceptions and experiences of students in online careers about the virtual environments they inhabit and transit, as well as their possibilities of agency and expression, and those of other subjects. Initially, we deepen into a theoretical systematization of virtual environments as social and educational spaces, whether they are institutional virtual classrooms or social media platforms. We also analyze data based on ad hoc quantitative data, and finally, we present a systematization of lines derived from the analysis for the construction of inclusive and democratic online educational spaces, which try not to repeat historical inequalities and contemplate the diversity of experiences and agencies. Methodologically, the production of quantitative data was combined with qualitative analysis based on a descriptive and analytical method. This work is based on a survey of students in online careers at the University (Nombre omitido).
\end{abstract}

Key-words: Educational virtual spaces. Social media. Online classrooms. Communications/Education. Agency.

\section{Referências}

BIANCHI, M.; SANDOVAL, L. Covid-19, desigualdad y reflexiones desde la periferia. Question/Cuestión, La Plata, v. 1, n. 1, jun. p. 347-347, 2020. Disponível em: https://perio.unlp.edu.ar/ojs/index.php/question/ article/view/6085. Acesso em: 21 junio 2020.

BUMACHAR, B. L.; FERREIRA, P. P. Materialidades e Maternidades: Agência distribuída e produção de copresença em redes espaçotemporais de cuidado mobilizadas por estrangeiras na Penitenciária Feminina da Capital (PFC)-SP. Interseções: Revista de Estudos 
Interdisciplinares, Rio de Janeiro, v. 20, n. 1, p. 67-84, 2018. Disponível em: https://www.e-publicacoes.uerj.br/index.php/intersecoes/article/ view/35864. Acesso em: 23 jun. 2020.

COUTO, E. S. Sexo além do sexo: performances corporais e pedagogias eróticas. Diversidade e Educação, Salvador, v. 3, n. 5, p. 10-18, 2015. Disponível em: https://periodicos.furg.br/divedu/article/view/6363. Acesso em: 21 jun. 2020.

DELFINO, S. Desigualdad y diferencia: retóricas de identidad en la crítica de la cultura. Revista Estudios, [s. l], CEA, v. 7, n. 18, 1998. Disponível em: https://dialnet.unirioja.es/servlet/ articulo? codigo $=5391643$. Acesso em: 24 jun. 2020 .

FICOSECO, V. Género y tecnologías digitales. La experiencia en entornos virtuales de aprendizaje. Buenos Aires: Editorial Universidad Nacional de Quilmes, 2018.

FICOSECO, V; CEVALLOS, G; OVIEDO, M. Influencia de la evaluación por pares y de la distribución visual de contenidos de cursos EaD en la valoración de los estudiantes. Educação Online, Rio de Janeiro, v. 14, n. 30, p. 17-31, 2019. Disponível em: http://educacaoonline.edu.puc-rio. br/index.php/eduonline/article/view/542. Acesso em 19 jun. 2020.

GAONA, M. Experiencia popular, ciudad e identidad en el noroeste argentino. La organización social Tupac Amaru. Oxford: Peter Lang Publishing, 2017.

KAPLÚN, G. Viral y vital. Ciudadanía, educación y comunicación. Question/Cuestión, La Plata v. 1, n. junio, p. 349-349, 2020. Disponível em: https://perio.unlp.edu.ar/ojs/index.php/question/article/ view/6129. Acesso em: 21 jun. 2020.

LEFEBVRE, H. La producción del espacio. Madrid: Capitán Swing, 2013.

MASSEY, D. For Space. Londres: SAGE Publications, 2005.

MINAYO, M. C. (org.). Pesquisa social: teoria, método e criatividade. Petrópolis: Vozes, 2011.

PRETTO, N. Educações, culturas e hackers: escritos e reflexões. Salvador: Edufba, 2017.

SAN MARTÍN, P.; GUARNIERI, G.; BONGIOVANI, P. Propuesta sociotecnológica para el desarrollo de repositorios de Acceso Abierto adecuados al contexto universitario argentino. E-Ciencias de la Información, Costa Rica, v. 4, n. 2, p. 1-26, 2014. Disponível em: https:// revistas.ucr.ac.cr/index.php/eciencias/article/view/15131. Acesso em: 21 jun. 2020.

SIBILIA, P. The Digitalization of Life. Dialogue and Universalism, [s. l], v. 29, n. 3, p. 93-102, 2019.

SILVEIRA, S. Democracia e os códigos invisíveis: como os algoritmos estão modulando comportamentos e escolhas políticas. São Paulo: Sesc, 2019. 
SOJA, E. Thirdspace. Journeys to Los Angeles and Other Real-andImagined Places. Massachusetts: Blackwell Published, 1996.

TURKLE, S. Alone Together: Why We Expect More from Technology and Less from Each Other. New York: Basic Books, 2011.

UNPA. Coronavirus: La UNPA se apoya en la virtualidad para enseñar durante la cuarentena | Universidad Nacional de la Patagonia Austral. Disponível em: https://www.unpa.edu.ar/noticia/coronavirus-la-unpase-apoya-en-la-virtualidad-para-ensenar-durante-la-cuarentena. Acesso em: 28 jun. 2020.

Submetido em: 20/07/2020

Aceito em: 28/10/2020 